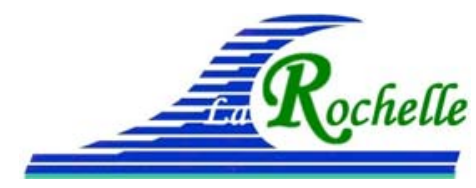

XV $V^{\text {èmes }}$ Journées Nationales Génie Côtier - Génie Civil

La Rochelle, 29 au 31 mai 2018

DOI:10.5150/jngcgc.2018.082 @ Editions Paralia CFL

disponible en ligne - http://www.paralia.fr - available online

\title{
Suivi des opérations d'immersion et système opérationnel pour la gestion des rejets de dragage, dans le cadre du projet d'extension en mer de l'Anse du Portier, Monaco
}

\section{Caroline TESSIER ${ }^{1}$, Camille JESTIN ${ }^{1}$, Meven HUIBAN ${ }^{1}$, Sina SAREMI ${ }^{\mathbf{1}}$, Aurélien GASC ${ }^{\mathbf{1}}$, Fabrice GOUAUD ${ }^{\mathbf{1}}$, Oriana Charles CASTELLANI ${ }^{2}$, Pauline WECH ${ }^{2}$}

\author{
1. DHI, 4 rue Edouard Nignon, 44300 Nantes, France. \\ cte@dhigroup.com \\ 2. Bouygues TP Monaco, 2 rue du Gabian, 98000 Monaco.
}

\section{Résumé :}

La Principauté de Monaco a lancé un nouveau projet d'urbanisation en mer s'intégrant harmonieusement à la façade littorale. Bouygues Travaux Public Monaco est en charge de la construction de l'Infrastructure Maritime, incluant en particulier des opérations de dragage sur site puis d'immersion en mer des sédiments dragués.

Au cours des études de conception, DHI a mis en place le modèle hydro-sédimentaire MIKE 3D afin de déterminer un mode opératoire optimisé pour les opérations d'immersion, dans le but d'en limiter les impacts environnementaux. L'étude a permis, sur la base de scenarii météo-océaniques identifiés, une optimisation des points de rejets et des méthodes d'immersion.

Pour la phase opérationnelle, la complexité des phénomènes en jeu et la sensibilité des résultats à la succession des scenarii météo-océaniques a mis en évidence la nécessité d'un outil de suivi des immersions tout au long des opérations, de juin 2017 à février 2018. Le modèle a également été exploité pour servir en tant qu'outil d'aide à la décision pour les opérations spécifiques de rejet à la Drague Aspiratrice en Marche. Deux modes d'exploitation ont ainsi été mis en œuvre :

- un mode "Hindcast", avec reproduction hebdomadaire des conditions hydrodynamiques et du devenir des sédiments immergés ;

- un mode "Forecast", avec des simulations journalières de prévision à $\mathrm{J}+1$ des conditions hydrodynamiques et des phénomènes de panaches turbides et de dépôts.

Ainsi une méthodologie complète a été développée et adaptée en fonction des exigences et contraintes du projet. Depuis la phase de conception jusqu'au suivi des opérations d'immersion, l'outil numérique exploité et développé tout au long du projet a permis d'aboutir à des modes opératoires optimisés, dans le respect des contraintes environnementales.

Mots-Clés : Rejets dragages, Système opérationnel, Modélisation hydro-sédimentaire, Monaco. 


\section{Thème 6 - Gestion durable des zones littorales et estuariennes}

\section{Introduction}

La principauté de Monaco a entrepris un projet d'extension en mer, au droit de l'Anse $\mathrm{du}$ Portier, s'intégrant harmonieusement à la façade littorale. Ce projet est particulièrement suivi et contraint d'un point de vue environnemental, du fait de la présence de réserves marines de part et d'autre du projet (Larvotto et Spélugues), d'espèces sensibles dans la zone littorale (herbiers de posidonies au Larvotto, coralligènes du tombant des Spélugues, les Grandes Nacres Pinna nobilis) et d'habitats sensibles plus au large (roches coralligènes localisées entre 60 et $80 \mathrm{~m}$ de fond; les zones sensibles profondes vers 80-120 m mises en évidence lors des campagnes RAMOGE (FOURT et al., 2015) et d'ANDROMEDE OCEANOLOGIE (2016). Le site Natura 2000 de Cap Martin à l'Est du territoire Monégasque est également assez proche. Bouygues TP MC est en charge de l'infrastructure maritime. Dans ce cadre, des opérations de dragages dans l'Anse du Portier et d'immersion des sédiments au large ont été réalisées par l'entreprise Jan de Nul de juin 2017 à février 2018.

La problématique du devenir des panaches turbides en mer constitue l'un des enjeux les plus importants du projet. Tout au long des études et des opérations, DHI a ainsi participé : à l'étude d'impact environnemental en phase d'AVant-Projet - AVP (en partenariat avec l'entreprise Tractebel Engineering), aux études complémentaires de la phase PROjet - PRO et enfin, à la phase d'EXEcution des opérations - EXE.

Cette communication a pour objectif de synthétiser les différentes stratégies de modélisation hydro-sédimentaires mises en place au vu des contraintes du projet et de son évolution, puis de comparer les résultats obtenus en phases de conception et d'exécution, en particulier sur le devenir des sédiments rejetés en mer.

\section{Le modèle et les stratégies de modélisation, selon les contraintes du projet}

\subsection{Le modèle hydro-sédimentaire du littoral Monégasque}

Le modèle hydrodynamique MIKE 3 FM (DHI, 2017) du littoral monégasque a été mis en place, sur la bande littorale, du Cap d'Antibes jusqu'à San Remo, et au large jusqu'à des profondeurs d'environ $2000 \mathrm{~m}$. Il couvre ainsi une zone d'environ $60 \mathrm{~km}$ de long par $28 \mathrm{~km}$ de large (figure 1). Le maillage flexible a une résolution de l'ordre de $1 \mathrm{~km}$ au large et est raffiné progressivement jusqu'à $10 \mathrm{~m}$ à la côte. La discrétisation verticale est de type "sigma-z" avec 60 niveaux verticaux.

Pour prendre en compte les interactions avec la circulation générale (courant ligure, effets de densité, tourbillons méso-échelle), le modèle est forcé aux limites marines par les résultats du modèle MENOR d'IFREMER (de résolution $1.2 \mathrm{~km}$ ) en courants, température et salinité (sorties PREVIMER puis sorties MARC; GARNIER et al. 2014 ; HERBERT et al. 2014). La marée a aussi été intégrée bien qu'elle soit d'amplitude faible. Le forçage par le vent et les flux de chaleur sont pris en compte en surface, à partir des données CFSR (Climate Forecast System Reanalysis) - puis 


\section{XVèmes Journées Nationales Génie Côtier - Génie Civil La Rochelle, 29 au 31 mai 2018}

d'ARPEGE de Météo-France. La fermeture turbulente est assurée par un modèle kepsilon, modifié pour prendre en compte les effets de flottabilité (Rodi 1980); il résout les équations de transport et de dissipation d'énergie cinétique, assurant une haute précision de mélange sur la colonne d'eau.

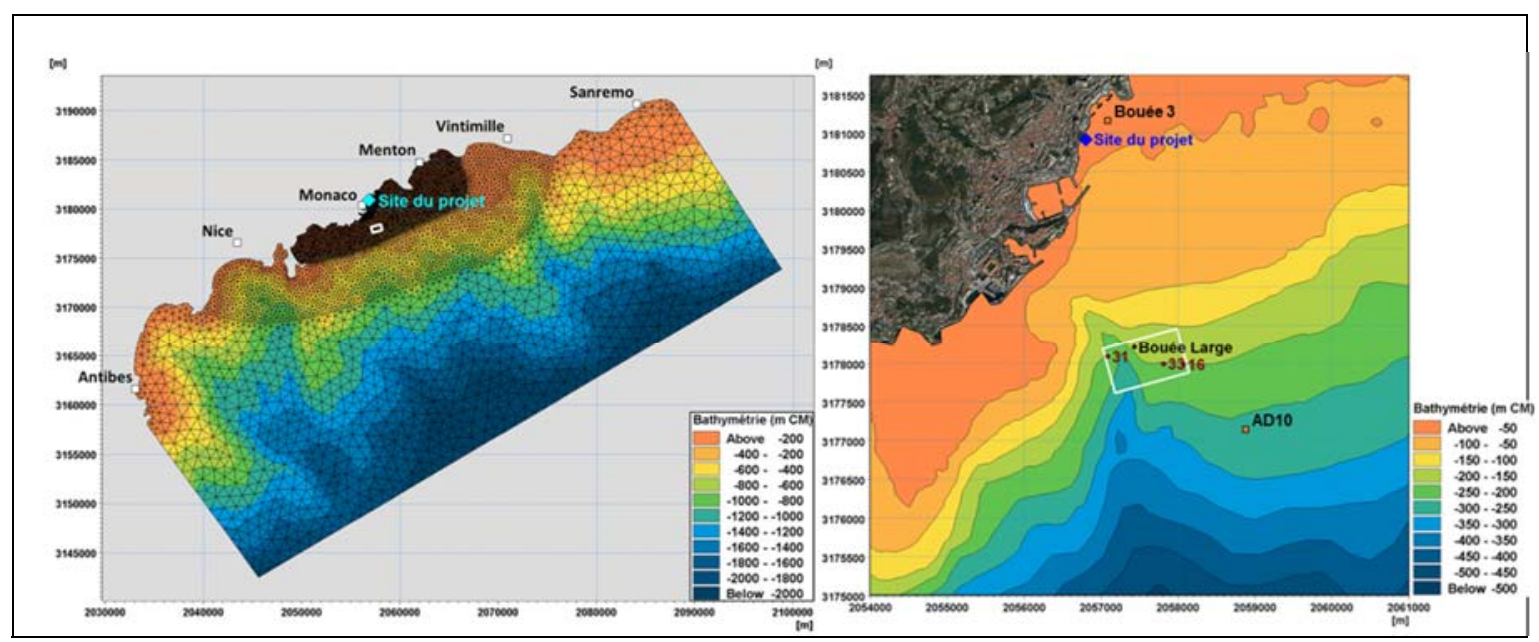

Figure 1. Emprise, maillage et bathymétrie du modèle MIKE 3D de Monaco (gauche). Localisation du site de projet, de la bouée et des points d'immersion des sédiments (droite).

Les simulations des panaches turbides induits par les opérations d'immersion ont été réalisées avec le module MT (Mud Transport) du modèle MIKE 3 FM. La dynamique des sédiments naturels n'a pas été considérée dans cette étude. Les processus d'érosiondépôt à l'interface eau-sédiment sont modélisés à partir des formulations classiques de PARTHENIADES (1965) et KRONE (1986). Deux fractions sédimentaires ont été prises en compte dans les simulations de dispersion des panaches turbides: la vase (avec ici un mode principal à $30 \mu \mathrm{m}$ ) et le sable fin (ici un mode à $90 \mu \mathrm{m}$ ), de manière à représenter la partie "fine" du spectre granulométrique des sédiments de l'Anse du Portier. Les sédiments plus grossiers sont considérés comme chutant rapidement au fond en étant moins dispersés que les particules fines. Le transport des particules fines dans la colonne d'eau est couplé à l'hydrodynamique et prend en compte les processus de floculation de manière simplifiée avec une vitesse de chute dépendante de la concentration en suspension.

Les opérations d'immersion modélisées concernent des clapages par chaland (depuis la surface) et des rejets à la Drague Aspiratrice en Marche - DAM, (à partir d'une élinde descendue à $75 \mathrm{~m}$ de profondeur). La chute convective des matériaux n'est pas modélisée directement et seule la libération dans la colonne d'eau d'une partie des sédiments rejetés est prise en compte dans les modélisations de dispersion des panaches turbides, avec le modèle précédemment décrit. Les flux sédimentaires libérés ("spill") pour les différentes méthodes d'immersion (clapages et DAM) sont estimés à partir de 


\section{Thème 6 - Gestion durable des zones littorales et estuariennes}

l'expérience de DHI, de celle de Jan de Nul et de la bibliographie (LAND \& BRAY, 2000 ; TRUITT, 1988 ; BECKER et al. 2015 ; AARNINKHOF \& LUIJENDIJK, 2010) mais s'appuient également sur les résultats de simulations complémentaires faites avec le module MIKE Disposal qui résout les équations de KOH \& CHANG (1973), comme le logiciel STFATE (1998), et qui permet de mieux connaître le comportement du panache turbide dans le champ proche.

Le comportement dynamique de la masse turbide au fond (après l'impact au fond et son effondrement dynamique, non modélisés) a été simulé de façon indépendante à partir d'un modèle haute résolution MIKE $3 \mathrm{FM}$, mis en place sur la zone d'immersion et prenant en compte les courants de densité liés aux fortes concentrations et à la bathymétrie du site. Les fortes concentrations et la pente du fond peuvent en effet amener les sédiments à voyager sur des distances importantes au fond. Les différents types d'opérations, avec les caractéristiques des sédiments associés (spectre granulométrique complet, teneur en eau), ont été simulés avec ce modèle et pris en compte dans les études.

\subsection{Modélisation de scenarii météo-océaniques réalistes}

Dans le cadre de l'étude d'impact environnemental, en phase AVP, mais également au cours des phases PRO et EXE, pour la mise à jour des dossiers d'autorisation, les modélisations des opérations de dragages et d'immersion ont été réalisées pour des conditions météo-océaniques types, représentatives de la climatologie aux abords de Monaco. Des scenarii types saisonniers ont ainsi été identifiés (chacun d'une durée de 3-4 jours), à partir des résultats du modèle régional MENOR sur les périodes 2001-2010 et 2014-2016.

Dans un premier temps, en accord avec la Principauté, c'est sur la période estivale qu'ont porté les études, saison critique en termes de qualité des eaux (saison balnéaire, faible renouvellement des eaux, croissance des posidonies). Quatre scenarii estivaux ont ainsi été identifiés en aout 2006 et septembre 2009, pour lesquelles des mesures de température, courants et vent étaient disponibles (SOGREAH, 2009).

L'effort de validation du modèle a porté sur la capacité du modèle à reproduire les différents processus en jeu et des événements réalistes types. Un événement d'upwelling côtier en saison estivale a pu ainsi être particulièrement bien reproduit, avec une remontée d'eau froide à la côte liée au vent d'Ouest (figure 2, gauche). Les faibles vitesses de courant en été sont bien reproduites jusqu'à $0.03 \mathrm{~m} / \mathrm{s}$. Les vents thermiques modulent ces faibles circulations à la côte. Le modèle a montré sa capacité à reproduire des cisaillements verticaux de courant induits par la stratification thermique et les gradients de densité grande échelle. Le modèle permet de prendre en compte également les interactions côte-large et les effets météorologiques qui génèrent des circulations tourbillonnaires méso-échelles. 


\section{XVèmes Journées Nationales Génie Côtier - Génie Civil La Rochelle, 29 au 31 mai 2018}

Du fait de l'évolution du planning des travaux, des méthodes de dragage et des volumes à draguer et immerger, les études ont été poursuivies en phase PRO et EXE, avec un besoin de réactivité face aux évolutions du projet. Les modélisations ont alors été réalisées successivement pour des scenarii printaniers, automnaux et hivernaux, identifiés sur la période de mai 2016 à janvier 2017. L'effort de validation du modèle a été poursuivi avec de nouvelles mesures de courantologie à la côte (figure 2, droite). La sensibilité au forçage vent a été étudiée (avec CFSR 0.2 , ARPEGE $0.1^{\circ}$ et AROME $0.025^{\circ}$ de Météo-France) : l'utilisation d'une donnée de vent de meilleure résolution permet éventuellement d'affiner la dynamique dans le modèle (en surface), mais ne modifie pas significativement les tendances (pics de courants, chute de température, ordres de grandeur), ce qui confirme la robustesse du modèle. A la côte, les effets topographiques ne sont pas bien pris en compte dans les modèles météorologiques et peuvent expliquer les écarts entre les courants modélisés et observés.

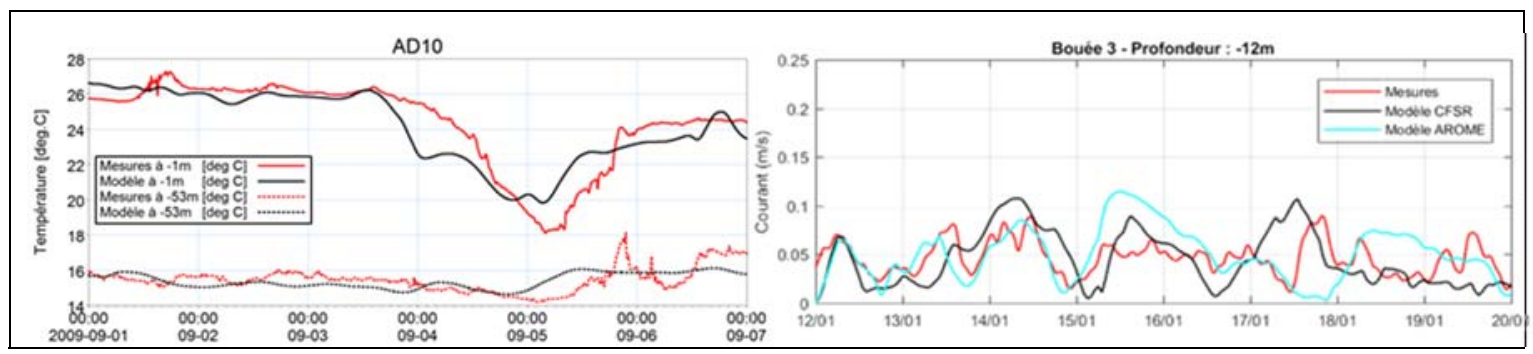

Figure 2. Calibration du modèle : températures mesurées et modélisées en surface et au fond en AD10 en septembre 2009 (gauche), courants mesurés et modélisés au fond à la bouée 3 avec différentes paramétrisations du vent en janvier 2017 (droite).

\subsection{Optimisation des points de rejets au large}

Avec l'objectif d'éviter le retour à la côte des sédiments rejetés au large et de minimiser la dispersion des panaches au large, les rejets en mer des sédiments issus des dragages ont été simulés pour les différents scenarii météo-océaniques, et pour différents points de rejet au large afin de déterminer les points "optimaux" pour chaque type d'opération, en tenant compte des contraintes environnementales : les roches profondes et la tête de canyon. Cet effort a permis d'identifier des secteurs proches du canyon, par des fonds de $200 \mathrm{~m}$ environ, mais suffisamment éloignés des zones sensibles, identifiées au préalable au cours de la phase PRO (ANDROMEDE OCEANOLOGIE, 2016). Le canyon s'est avéré être une zone très peu sensible avec des sédiments vaseux déjà en place et très peu peuplés.

Les simulations numériques ont aussi montré que rejeter plus au large (au-delà de l'isobathe $250 \mathrm{~m}$ ) induisait une dispersion plus forte des panaches du fait d'un comportement passif alors atteint rapidement dans la colonne d'eau. De plus, augmenter la profondeur de rejet de la DAM amplifiait également la dispersion du fait des courants 


\section{Thème 6 - Gestion durable des zones littorales et estuariennes}

de jet horizontaux générés au fond lors de l'impact. Ainsi, le choix de rejeter à $75 \mathrm{~m}$ de fond une mixture eau-sédiment par des fonds de $200 \mathrm{~m}$, avec une vitesse envisagée en sortie d'élinde de $4.1 \mathrm{~m} / \mathrm{s}$, permet de limiter les impacts du fait que le panache atteint presque sa profondeur d'équilibre au fond.

Finalement, les modélisations ont permis d'identifier des points de rejets spécifiques à chaque type d'opération (méthode d'immersion et nature du matériau immergé), mais indépendants des conditions météo-océaniques : les points R16, R31 et R33 (figure 1). Les contraintes d'exécution des travaux ont ainsi été grandement allégées. Pour estimer l'impact de toutes les opérations d'immersion, en termes de dépôts accumulés au large, les résultats des simulations des scenarii météo-océaniques individuels ont été cumulés et pondérés en fonction de leur représentativité dans la climatologie, et de façon à considérer le volume total immergé pour chaque opération.

\subsection{Modélisations "Hindcast" et "Forecast" en phase opérationnelle}

Les immersions en mer se sont étendues de juin 2017 à février 2018, avec trois phases principales :

a) Les opérations de dragages des sédiments grossiers provenant des remblais anthropiques ont été réalisés de juin à octobre 2017 avec des clapages au point R16(environ $15 \%$ de la totalité des sédiments immergés). Ces sédiments avaient une teneur en fines estimée à $5 \%$ de vases et $10 \%$ de sables fins.

b) Les opérations à la DAM ont eu lieu d'octobre 2017 à février 2018 (environ $80 \%$ de la totalité des sédiments immergés), avec des rejets par élinde à $75 \mathrm{~m}$, en R31, de sédiments vaseux ( $40 \%$ de vases et $37 \%$ de sables fins).

c) Les opérations de clapages des vases du Larvotto (environ 5\% de la totalité des sédiments immergés) ont été réalisées, en R33, courant février 2018. Finalement, au vu de la faible quantité concernée, c'est un rejet en surface d'une mixture eausédiment qui a été réalisé, du fait d'un dragage à la DAM au lieu d'un dragage mécanique prévu initialement.

Pour assurer le suivi des opérations d'immersion, les simulations "Hindcast" des opérations réellement réalisées ont été entreprises dès le début de la phase EXE, en simulant chaque semaine les opérations réalisées (avec un décalage de deux semaines environ), sur la base des rapports d'immersion fournis par Bouygues TP MC. Ces simulations sont réalisées en assurant la continuité des événements météo-océaniques et des dépôts accumulés chaque semaine. Le forçage par le vent et les flux de chaleur sont issus des prévisions journalières du modèle ARPEGE $0.1^{\circ}$ de Météo-France. Les conditions limites marines ont été extraites des résultats du modèle MENOR d'IFREMER, à partir des meilleures estimations mises à jour chaque semaine avec un délai d'une semaine.

Les simulations haute résolution du devenir de la masse turbide au fond ont également été simulées chaque semaine, tout au long de la période d'immersion. 


\section{XVèmes Journées Nationales Génie Côtier - Génie Civil La Rochelle, 29 au 31 mai 2018}

Enfin, devant la variabilité interannuelle des courants au large, un système de prévision journalière ("Forecast") des courants et des dépôts induits par les rejets a été mis en place (avec MIKE OPERATIONS) pour les opérations à la DAM au point R31. Ces opérations pouvant mener à une dispersion importante des panaches dans la colonne d'eau, il était en effet important d'anticiper les risques en fonction des conditions météo-océaniques prévues, et de disposer ainsi d'un outil d'aide à la décision. L'accumulation de certaines conditions défavorables pouvant mener à des situations critiques, le système a permis d'assurer la faisabilité des opérations d'immersion, jour après jour pour toute la durée des opérations à la DAM en R31. L'équipe Travaux, à bord de la DAM, avait accès à une interface Web présentant les résultats des simulations et permettant de paramétrer chaque soir les opérations prévues le lendemain, et simulées chaque veille au soir.

\section{Les courants sur le site d'immersion}

Les résultats des simulations du modèle Monaco ont été confrontés aux mesures in-situ de courants réalisées au large de mai 2017 à février 2018 (Signature 250 Nortek mis en place sur une bouée en surface par NortekMed, pour Jan de Nul et Bouygues TP MC). Le point de mesure était situé entre les points d'immersion R33 et R16, par des fonds de $180 \mathrm{~m}$.

De façon générale, les simulations ont montré une très bonne reproduction des courants dominants, portant vers le Sud-Ouest et le Nord-Est, avec une petite rotation dans le bas de la colonne d'eau à l'Ouest/Sud-Ouest et l'Est/Nord-Est. Les vitesses des courants modélisés sont légèrement plus fortes que dans les mesures en surface lors des événements météorologiques intenses, mais ils sont plus faibles dans les couches de fond. Les mesures présentent cependant une dispersion et une variabilité assez fortes, liées parfois à des perturbations des signaux qui restent très bruités dans le bas de la colonne d'eau.

De plus, les simulations reproduisent très bien la structure verticale des courants avec une dynamique visible jusqu'à environ $50 \mathrm{~m}$ de profondeur en saison estivale, reliée à la forte stratification thermique, et qui s'approfondit à l'automne jusqu'à environ $100 \mathrm{~m}$, avec la disparition de cette stratification.

La comparaison des roses de courants mesurés et modélisés est présentée en figure 3 pour la période automne-hiver, pendant laquelle les rejets à la DAM ont eu lieu, et qui sont les opérations les plus sensibles à la succession des événements météo-océaniques. Les résultats des simulations Hindcast et Forecast sont assez proches, avec cependant dans le Forecast, une composante Nord-Est plus marquée en sub-surface et à miprofondeur, alors qu'au fond les courants vers le Sud-Ouest apparaissent un peu plus fréquents. Les roses associées aux quatre scenarii automnaux, pondérés de leur représentativité sur la durée des rejets à la DAM, mettent en évidence une contribution plus marquée des événements portant au Nord-Est dans le bas de la colonne d'eau. 


\section{Thème 6 - Gestion durable des zones littorales et estuariennes}

L'analyse a montré en effet que certains scenarii automnaux incluent des périodes de faible circulation vers l'Est-Nord-Est, à la suite d'un événement plus intense vers le Sud-Ouest. Ces résultats mettent en évidence l'importance de la durée des événements même s'ils sont d'intensité faible, pour le devenir des panaches turbides (voir section 4).

\section{Le devenir des rejets de dragages en mer}

Les simulations de dispersion des panaches turbides dans la colonne d'eau ont permis d'estimer des cartes de dépôts des sédiments fins rejetés lors des opérations d'immersion. Les panaches turbides sont transportés d'un côté ou de l'autre des points de rejet, et plus ou moins loin, en fonction des événements météo-océaniques ayant lieu au moment et dans les heures qui suivent les opérations. En général, le panache d'un rejet est suffisamment dispersé en une journée (concentration en suspension inférieure à $1 \mathrm{mg} / \mathrm{L}$ ). Mais la répétition des opérations alimente en quasi continu la colonne d'eau en sédiments, ce qui rend sensibles les résultats de dépôts à la succession des événements et aux périodes de courants faibles.

Les hypothèses de "spill" ont été définies pour chaque opération, sur la base des expertises de DHI et Jan de Nul, et des résultats de simulation du module MIKE Disposal :

a) Les clapages de matériau grossier en R16 ont été simulés en considérant un spill de $15 \%$ des fines dans la colonne d'eau et le reste dans la couche de fond, la chute des matériaux denses étant très rapide au fond.

b) Les rejets à la DAM ont été simulés en considérant un spill de $10 \%$ des sédiments, répartis entre l'élinde $(-75 \mathrm{~m})$ et le fond, et le reste dans la couche de fond, la masse turbide chutant rapidement sous l'effet de la gravité au fond. Cette hypothèse est conservative car la masse turbide au fond subit en fait son comportement propre d'écoulement sur la pente, ce qui a été modélisé par le modèle haute résolution. Seule une partie de cette masse est donc susceptible d'être dispersée par les courants près du fond. Les résultats aux points exacts des rejets ne sont donc pas exploités, c'est essentiellement les risques de dispersion lointaine qui sont évalués par les cartes de dépôts. De plus, l'hypothèse de spill au cours de la descente du jet a été réduite à 5\% pour les modélisations Hindcast et Forecast, car la vitesse de chute en sortie d'élinde a finalement été augmentée à $7 \mathrm{~m} / \mathrm{s}$ au lieu de $4.1 \mathrm{~m} / \mathrm{s}$ prévue initialement.

c) Les immersions des vases du Larvotto en R33 ont finalement été réalisées par clapage d'une mixture eau-sédiment à partir de la surface. Les hypothèses de spill, considérées en phase PRO à $15 \%$ dans la colonne d'eau, ont donc été modifiées pour considérer en plus une libération de $15 \%$ des particules de vases en surface. Le reste est injecté dans les $50 \mathrm{~m}$ au-dessus du fond, diamètre estimé du panache lorsqu'il touche le fond.

Les cartes de dépôts accumulés de toutes les opérations sont présentées en figure 4 pour les résultats: du Hindcast, du Forecast et des scenarii types, pondérés de leur 


\section{XVèmes Journées Nationales Génie Côtier - Génie Civil \\ La Rochelle, 29 au 31 mai 2018}

représentativité pour chaque type d'opération prévue en phase de conception. Pour ce dernier cas, la carte des dépôts cumulés apparait cohérente à celle du Hindcast avec cependant une étendue plus importante vers le Nord-Est des dépôts. L'opération des rejets à la DAM en R31 est en effet particulièrement sensible aux événements rencontrés et à leur succession. Or les scenarii automnaux identifiés présentent en effet des courants plus fréquents vers le Nord-Est que les conditions réelles rencontrées au cours de l'automne-hiver 2017-2018. A cela s'ajoute le fait que les caractéristiques des opérations ont été sensiblement modifiées en phase d'exécution, avec un rendement plus faible qu'envisagé (des rejets en quantité plus faible mais sur une durée plus longue) du fait de contraintes plus fortes sur les dragages (difficulté de mise en œuvre, arrêts dus aux conditions météorologiques, etc.). Les hypothèses de spill ont aussi été réduites. Aussi, les simulations Hindcast montrent finalement une étendue moins importante des dépôts que celle estimée en phase de conception. Les résultats du Forecast apparaissent assez proches de ceux du Hindcast, avec une dispersion légèrement plus faible vers le Sud-Ouest des rejets. Ces résultats sont très satisfaisants, au vu des imprécisions possibles sur les prévisions des forçages météorologiques et les conditions limites marines, particulièrement délicates à modéliser en Méditerranée. Le choix du site d'immersion a permis également de bénéficier des effets bathymétriques sur la dispersion des panaches, les dépôts tendent ainsi à suivre les isobathes et/ou suivre la pente du canyon. Les zones sensibles situées au Nord-Ouest, par des fonds de 80-100 m ne sont donc pas impactées.

\section{Conclusion}

La modélisation du devenir des rejets en mer issus des dragages des sédiments de l'Anse du Portier a été menée dans un premier temps à partir de scenarii météoocéaniques types, afin d'assurer la bonne reproduction des processus en jeu en Méditerranée, et de manière à identifier rapidement des points d'immersion pertinents limitant les impacts potentiels des rejets. L'utilisation de scenarii types a permis une forte réactivité face aux contraintes réglementaires et aux évolutions du projet (planning, méthodes, volumes dragués, etc.). Malgré la variabilité interannuelle des événements météo-océaniques saisonniers, les directions dominantes des circulations sont bien reproduites et les tendances à la dispersion des panaches, contraintes également par la bathymétrie du site, sont cohérentes avec les résultats du Hindcast réalisé en phase d'exécution. Il est cependant apparu un écart notable sur la dispersion vers le Nord-Est finalement moins marquée que prévu pour les rejets à la DAM, du fait de courants moins fréquents dans cette direction au cours de l'automne-hiver 20172018. Mais les écarts entre les résultats obtenus en phase étude et en phase de travaux sont surtout liés à des conditions d'opérations qui ont changé. L'étude a ainsi montré l'importance d'adapter rapidement les hypothèses de modélisation en fonction de l'évolution des méthodes de dragages utilisées et des contraintes du projet, en 


\section{Thème 6 - Gestion durable des zones littorales et estuariennes}

compétition permanente avec les enjeux environnementaux à respecter (disponibilité et mise en œuvre des dragues, planning à tenir, accessibilité et fréquentation du site, etc.). La mise en place de simulations Hindcast est donc très pertinente pour ce type de projet à forte évolution. Elles permettent d'assurer un suivi régulier (toutes les semaines) de l'impact des opérations réellement réalisées. Une instrumentation complémentaire sur site aurait été intéressante mais difficilement réalisable (suivi des courants, de la turbidité et des dépôts en plusieurs points autour de la zone d'immersion). De plus, les levés bathymétriques intermédiaires et finaux n'ont pas permis d'obtenir une précision inférieure à $0.50 \mathrm{~m}$ à ces profondeurs de plus de $200 \mathrm{~m}$. La modélisation reste donc le meilleur outil pour l'estimation des faibles dépôts induits par ces rejets en mer.

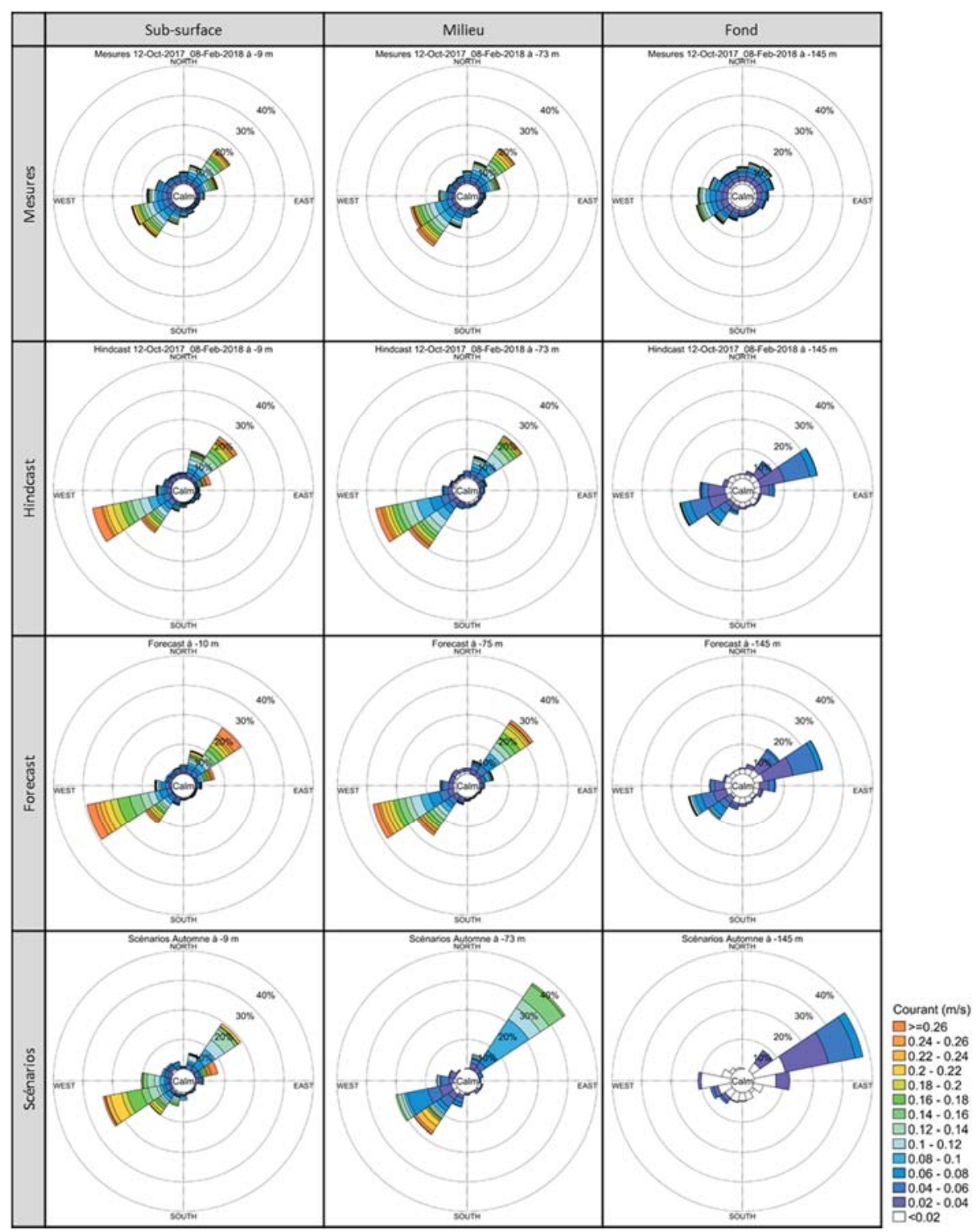

Figure 3. Roses des courants mesurés et modélisés (issus du Hindcast, du Forecast et des scenarii automnaux) de mi-octobre 2017 à début février 2018. 


\section{XVèmes Journées Nationales Génie Côtier - Génie Civil \\ La Rochelle, 29 au 31 mai 2018}

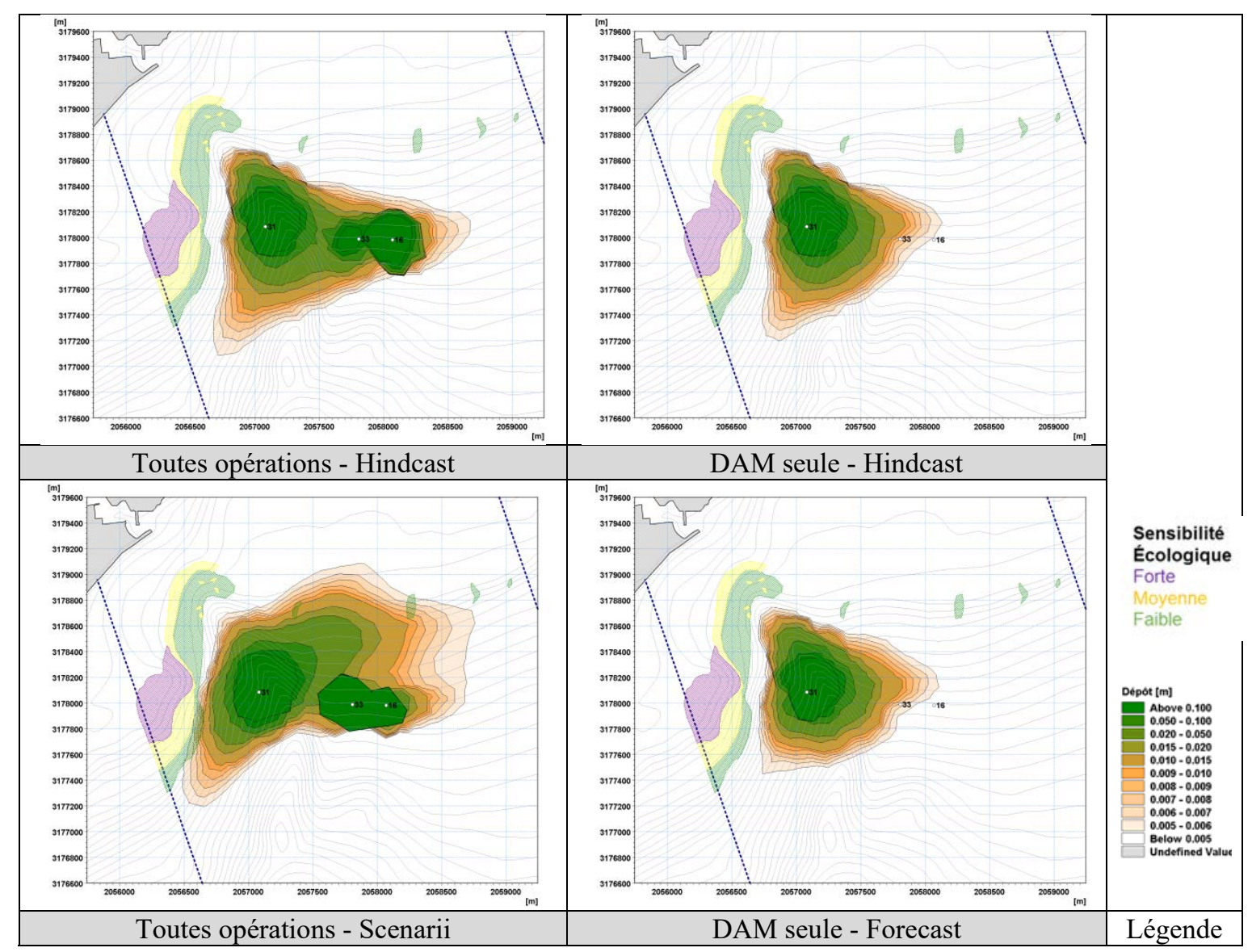

Figure 4. Cartes des dépôts accumulés liés à la dispersion des panaches turbides modélisés (issus du Hindcast, du Forecast et des scenarii considérés en phase PRO).

Enfin, le système opérationnel de prévisions (Forecast) des opérations d'immersion à la DAM a été nécessaire pour garantir à la Principauté la faisabilité de ce type d'opération, dont les impacts potentiels sont en effet sensibles à la succession des événements météo-océaniques. Les résultats du Forecast ont de plus été confirmés par ceux du Hindcast, en termes de cartes de dépôts cumulés.

Depuis la phase de conception jusqu'au suivi des opérations d'immersion, l'outil numérique exploité et développé tout au long du projet a ainsi permis d'aboutir à des modes opératoires optimisés, dans le respect des contraintes environnementales.

\section{Références Bibliographiques}

AARNINKHOF S., LUIJENDIJK A. (2010). Safe disposal of dredged material in a sensitive environment based on innovative plume predictions. Revue TERRA \& AQUA, $\mathrm{n}^{\circ} 119$, juin 2010.

ANDROMEDE OCEANOLOGIE (2016), Reconnaissances complémentaires de la zone d'immersion, Qualité du Benthos de substrat dur (ROV) \& Qualité des eaux. Rapport d'étude INF-PRO-AND-ENV-TTZ-GEN-RAP-275006. 


\section{Thème 6 - Gestion durable des zones littorales et estuariennes}

SOGREAH (2009). Actualisation de l'état zero du milieu littoral - Campagne de mesures 2009, rapport d'étude $\mathrm{n}^{\circ} 1741485$, mai 2010, pour la Principauté de Monaco, Direction de la Prospective, de l'Environnement et de la Mobilité.

BECKER J., VAN EEKELEN E., VAN WIECHEN J., DE LANGE W., DAMSMA T., SMOLDERS T., VAN KONINGSVELD M. (2015). Estimating source terms for far field dredge plume modelling. Journal of Environmental Management, Vol. 149, pp. 282-293. https://doi.org/10.1016/j.jenvman.2014.10.022

DHI (2017), MIKE 21 \& MIKE 3 Flow Model FM - Hydrodynamic and Transport Module - Scientific Documentation, 59 p.

URL : http://manuals.mikepoweredbydhi.help/2017/Coast_and_Sea/MIKE_321_FM_Scientific_Doc.pdf

FOURT M., GOUJARD A., CANESE S.P., SALVATI E., TUNESI L., DANIEL B., VISSIO A. (2015), Rapport de la campagne océanographique "RAMOGE Exploration canyon et roches profondes 2015". Accord RAMOGE - Agence des Aires Marines Protégées, $81 \mathrm{p}$.

GARNIER V., PAIRAUD Y., NICOLlE A., ALEKSEENKO E., BAKLOUTI M., THOUVENIN B., LECORNU F., GARREAU P. (2014). MENOR, a high resolution $(1.2 \mathrm{~km})$ modelling of the North-Western Mediterranean Sea routinely run by the PREVIMER operational forecast system, 2014-04 Mercator ocean - Quaterly Newsletter.

HERBERT G., GARREAU P. GARNIER V., DUMAS F., CAILLAUD S., CHARNUT J., LEVIER B., AZNAR R. (2014). Downscaling from Oceanic Global Circulation model towards regional and coastal model using spectral nudging techniques: application to Mediterranean Sea and IBI area models, 2014-04 Mercator oceanQuaterly Newsletter.

KOH R.C., CHANG Y.C. (1973). Mathematical model for barged ocean disposal of wastes. Environmental Protection Agency Series EPA 660/2-73-029, December 1973 [Office of Research and Development, US Environmental Protection Agency].

KRONE B. (1986). The significance of aggregate properties to transport processes. In : Mehta A.J. (Ed) Estuarine Cohesive Sediment Dynamics, Springer Verlag, pp. 66-84.

LAND J.M., BRAY R.N. (2000). Acoustic measurement of suspended solids for monitoring of dredging and dredged material disposal. Journal of Dredging Engineering, Vol. 2(3), pp. 1-17.

PARTHENIADES E. (1965). Erosion and deposition of cohesive soils. Journal of the Hydraulics Division, Proceedings of the ASCE 91 (HY1), 105-139.

STFATE (1998). Evaluation of dredged material proposed for discharge in waters of the U.S. - Testing Manual. Environmental Protection Agency report 823-B-98-004, Appendix C2.0, February 1998 [Office of Water, US Environmental Protection Agency $\&$ Department of the Army, US Army Corps of Engineers].

TRUITT C. L. (1988). Dredged material behavior during open-water disposal. Journal of Coastal Research, Vol. 4(3), pp. 389-397. 